\title{
Cardiac biomarkers and long-term outcomes of exacerbations of COPD: a long-term follow-up of two cohorts
}

\author{
Eskandarain Shafuddin ${ }^{1,3}$, Sarah M. Fairweather ${ }^{1,3}$, Catherina L. Chang ${ }^{1}$, \\ Christine Tuffery ${ }^{1}$ and Robert J. Hancox (10 ${ }^{1,2}$
}

Affiliations: ${ }^{1}$ Dept of Respiratory Medicine, Waikato Hospital, Hamilton, New Zealand. ${ }^{2}$ Dept of Preventive and Social Medicine, Otago Medical School, University of Otago, Dunedin, New Zealand. ${ }^{3}$ These authors contributed equally.

Correspondence: Eskandarain Shafuddin, Dept of Respiratory Medicine, Waikato Hospital, Pembroke Street, Hamilton 3206, New Zealand. E-mail: eskandarain.shafuddinawaikatodhb.health.nz

\section{ABSTRACT}

Background: COPD patients often have cardiac comorbidities. Cardiac involvement at the time of a COPD exacerbation is associated with a high short-term mortality, but whether this influences long-term outcomes is unknown. We explored whether biomarkers of cardiac dysfunction at the time of a COPD exacerbation predict long-term outcomes.

Methods: Two prospective cohorts of patients admitted to Waikato Hospital for exacerbations of COPD were recruited during 2006-2007 and 2012-2013. N-terminal pro-B-type natriuretic peptide (NT-proBNP) and troponin $\mathrm{T}$ were measured on admission and were used to indicate cardiac stretch and myocardial injury, respectively. 5-year survival after discharge and subsequent admissions for cardiac disease and COPD exacerbations were analysed using Kaplan-Meier and Cox proportional hazards tests.

Results: The overall 5-year mortality was 61\%. Patients with high NT-proBNP on admission had higher mortality than those with normal cardiac biomarkers (adjusted hazard ratio (aHR) 1.76, 95\% CI 1.18-2.62). High NT-proBNP was also associated with a higher risk of future cardiac admissions (aHR 1.75, 95\% CI 1.2-2.55). Troponin T levels were not associated with long-term survival (aHR 0.86, 95\% CI $0.40-1.83$ ) or future cardiac admissions (aHR 0.74, 95\% CI 0.34-1.57). Neither biomarker predicted future COPD exacerbations.

Conclusion: The long-term prognosis following a hospitalisation for an exacerbation of COPD is poor with less than half of patients surviving for 5 years. Elevated NT-proBNP at the time of a COPD exacerbation is associated with higher long-term mortality and a greater likelihood of future cardiac admissions, but not future COPD exacerbations.

$@$ ERSpublications

Elevated NT-proBNP at the time of \#AECOPD is associated with higher long-term mortality and a greater likelihood of future cardiac admissions, but not future COPD exacerbations https://bit.ly/37wnRQP

Cite this article as: Shafuddin E, Fairweather SM, Chang CL, et al. Cardiac biomarkers and longterm outcomes of exacerbations of COPD: a long-term follow-up of two cohorts. ERJ Open Res 2021; 7: 00531-2020 [https://doi.org/10.1183/23120541.00531-2020].

This article has supplementary material available from openres.ersjournals.com

Received: 28 July 2020 | Accepted: 26 Nov 2020

Copyright $\odot$ ERS 2021. This article is open access and distributed under the terms of the Creative Commons Attribution Non-Commercial Licence 4.0. 


\section{Introduction}

Cardiovascular disease is an important comorbidity in COPD patients and is estimated to cause up to $37 \%$ of deaths $[1,2]$. People with COPD have a high risk of cardiovascular disease because of the shared risk factors of advancing age and smoking, which contribute to the pathophysiology of both conditions [3]. However, lung and cardiovascular disease frequently coexist even in nonsmokers, indicating that there are other mechanisms linking these diseases.

Cardiac comorbidities are particularly important in the setting of acute exacerbations of COPD. Several studies have documented high rates of myocardial infarction, arrhythmias and heart failure among patients experiencing exacerbations of COPD, and these are associated with a worse short-term prognosis [4-9]. Exacerbations of COPD, heart failure and acute coronary syndromes can all present as dyspnoea or chest discomfort, and it is likely that cardiovascular disease is underdiagnosed in patients with COPD exacerbations leading to undertreatment [4].

We have previously reported on two prospective observational cohort studies of biochemical evidence of cardiac pathology in patients admitted to hospital for acute exacerbations of COPD without clinical evidence of acute cardiac disease [10-13]. The first study (cohort 1) found that 28\% of participants had elevated N-terminal pro-B-type natriuretic peptide (NT-proBNP) levels and $17 \%$ had elevated troponin $\mathrm{T}$ [10]. Elevated cardiac biomarkers were associated with substantially higher 30-day mortality. These findings were confirmed in the second study (cohort 2) [12]. These biomarkers appear to indicate acute or chronic cardiac involvement that cannot be reliably detected by clinical assessment, routine chest radiographs or electrocardiograms [13]. Furthermore, the elevated cardiac biomarkers are associated with both left and right heart dysfunction [14]. Thus, cardiac involvement has at least short-term prognostic significance in the setting of COPD exacerbations.

The long-term prognosis after hospitalisation for an exacerbation of COPD is poor, with a 5-year mortality of around $60 \%$ [4]. It is unknown to what extent subclinical cardiac involvement influences this prognosis beyond the acute phase of the COPD exacerbation.

To test the hypothesis that elevated biomarkers of cardiac injury (troponin) and cardiac stretch (NT-proBNP) during exacerbations of COPD would predict long-term prognosis for survival, further COPD exacerbations and future cardiac events, we combined and analysed the data from these two prospective cohorts.

\section{Methods \\ Study participants}

Two cohorts of consecutive patients admitted to Waikato Hospital for exacerbations of COPD were recruited (cohort 1, n=247 (2006-2007) and cohort 2, n=176 (2012-2013)). The details of these cohorts have been published previously $[10,11]$. Each cohort recruited patients over 1 complete year. Patients who did not live locally or could not attend follow-up were excluded. Both cohorts recruited all consenting patients with clinically diagnosed exacerbations of COPD but excluding those with suspected acute cardiac disease and those with pneumonia (consolidation on chest radiographs). The second cohort also excluded patients with end-stage renal disease. For those with multiple admissions over the year, the first admission was regarded as the index admission.

All participants gave written consent at the time of study inclusion, and approval was obtained for both cohorts from the New Zealand Health and Disability Ethics Committee.

\section{Data collection}

For this analysis the electronic records of patients were accessed to ascertain mortality within 5 years of discharge from hospital. We also recorded readmissions for exacerbations of COPD and cardiac admissions with heart failure or acute coronary syndromes within the Waikato District Health Board region. Fourteen participants recruited in both cohort 1 and 2 were removed from cohort 2 to avoid duplication.

\section{Cardiac biomarkers}

Both cohorts collected blood for measurement of NT-proBNP and cardiac troponin $\mathrm{T}$ within $24 \mathrm{~h}$ of admission to hospital. Measurements of NT-proBNP and troponin T were performed by Waikato Hospital laboratory using quantitative electrochemiluminescence assay. NT-proBNP levels in both cohorts had a detection limit of $0.6 \mathrm{pmol} \cdot \mathrm{L}^{-1}$ (Electsys proBNP, Roche Diagnostics Corporation). The troponin detection limit was $0.01 \mu \mathrm{g} \cdot \mathrm{L}^{-1}$ for cohort 1 and $5 \mathrm{ng} \cdot \mathrm{L}^{-1}$ for cohort 2 , because a high sensitivity troponin assay was introduced between the cohorts (troponin and TnT-hs, Roche Diagnostics Corporation).

NT-proBNP $>220 \mathrm{pmol} \cdot \mathrm{L}^{-1}$ and troponin $\mathrm{T}>0.03 \mu \mathrm{g} \cdot \mathrm{L}^{-1}$ in cohort 1 and $>50 \mathrm{ng} \cdot \mathrm{L}^{-1}$ in cohort 2 are considered abnormal according to local laboratory reference values. These levels were used to group 
participants into normal cardiac biomarkers, high troponin T only, high NT-proBNP only and both high troponin T and NT-proBNP groups (figure 1).

\section{Statistical analysis}

The primary end point was survival after discharge. Patients who died during their index admission were excluded from the analyses. This was plotted using Kaplan-Meier survival curves for the groups with normal or abnormal biomarkers. Cox proportional hazard ratios (HRs) were calculated with and without adjustment for acute COPD severity using the CURB-65 (Confusion, Urea, Respiratory rate, Blood pressure, Age $\geqslant 65$ ) score and acidaemia (blood $\mathrm{pH}<7.30$ ) on admission, and severity of airflow obstruction using forced expiratory volume in $1 \mathrm{~s}\left(\mathrm{FEV}_{1} \%\right.$ pred) $[10,12]$. Secondary end-points were readmissions for exacerbations of COPD or acute cardiac disease. Because patients may have died before readmission, these were calculated as COPD admission-free survival and cardiac admission-free survival, respectively, with follow-up censored at the date of death. Because these analyses used pre-existing cohorts, no formal sample size calculations were undertaken. Analyses were conducted with Stata (v.16; StataCorp, College Station, TX, USA).

\section{Results}

The contribution of each cohort is shown in figure 1. The demographic and clinical characteristics of the participants are shown in table 1 . Detailed baseline characteristics have been published previously [10, 11]

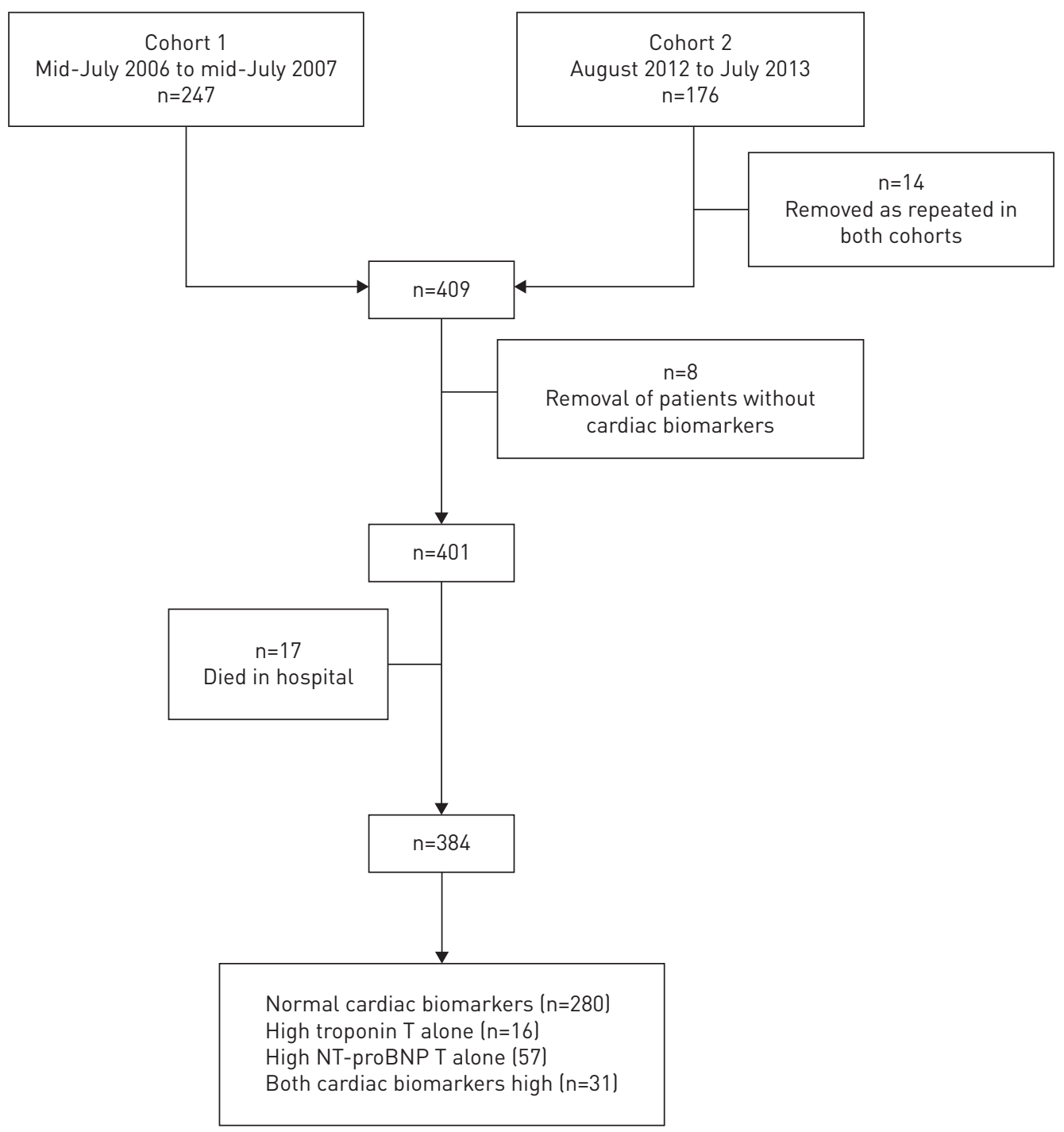

FIGURE 1 Study design. Patients who died in hospital $(n=17)$ were included in overall mortality data but were excluded from survival analyses after discharge. NT-proBNP: N-terminal pro-B-type natriuretic peptide. 
and are available in tables S1 and S2. Including those who died during the index admission, 7\%, 33\% and $61 \%$ of patients died in the first 30 days, 2 years and 5 years, respectively (figure 2). Risk factors for death at 5 years after index admission are shown in table S3 and these include a history of cardiac disease recorded at the time of the index admission.

Kaplan-Meier survival curves for those who survived to discharge from the index admission ( $\mathrm{n}=384)$ for each cardiac biomarker group are shown in (figure 2). Visual inspection indicated that the proportional hazards assumption was met, and the Cox proportional HRs are shown in table 2. Compared to participants who had normal cardiac biomarker measurements, participants with elevated NT-proBNP only had 2.13 times higher mortality during this period. This remained the case after adjusting for exacerbation severity (acidaemia and CURB-65) and baseline lung function ( $\mathrm{FEV}_{1} \%$ pred) (table 2). By contrast elevated troponin $\mathrm{T}$ only in participants was not associated with a worse 5 -year survival compared to those with normal biomarkers, and participants with abnormalities of both NT-proBNP and troponin T had similar survival to those who only had abnormal NT-proBNP (figure 2, table 2). These findings were similar after adjusting for a history of cardiac disease (table S4).

Cardiac biomarkers were not associated with future COPD exacerbations (figure 3). However, compared to participants who had normal cardiac biomarker measurements, participants with elevated NT-proBNP alone or both elevated NT-proBNP and troponin T were more likely to be admitted for cardiac disease within 5 years (figure 4). Elevated troponin $\mathrm{T}$ alone was not associated with future cardiac admissions.

\section{Discussion}

We have found that an elevated NT-proBNP is a strong predictor of poor long-term prognosis among patients admitted to hospital with an exacerbation of COPD. The overall mortality rate was nearly twice as high among those with elevated NT-proBNP even after adjusting for lung function and exacerbation

\section{TABLE 1 Baseline characteristics}

\begin{tabular}{|c|c|c|}
\hline Characteristic & Subjects & Value \\
\hline Age years & 409 & $70.1(69-71.2)$ \\
\hline BMI $\mathrm{kg} \cdot \mathrm{m}^{-2}$ & 401 & $24.6(23.9-25.2)$ \\
\hline Current smoker & 409 & $126(31)$ \\
\hline $\mathrm{FEV}_{1} \mathrm{~L}$ & 404 & $0.79(0.76-0.83)$ \\
\hline FEV $1 \%$ pred & 404 & $34.7(33.3-36.2)$ \\
\hline Acidaemia" & 364 & $56(15)$ \\
\hline$P_{\mathrm{aCO}_{2}} \mathrm{mmHg}$ & 315 & $47.9(46.3-49.7)$ \\
\hline$P_{\mathrm{aO}_{2}} \mathrm{mmHg}$ & 312 & $63.8(61.5-66.2)$ \\
\hline History of cardiac disease & 409 & 152 (37) \\
\hline History of malignancy & 409 & $42(10)$ \\
\hline \multicolumn{3}{|l|}{ Cardiac medication on admission } \\
\hline$\beta$-blocker & 409 & $45(11)$ \\
\hline Calcium-channel blocker & 409 & $97(24)$ \\
\hline Angiotensin-converting enzyme inhibitor/angiotensin receptor blocker & 409 & $158(39)$ \\
\hline Diuretic & 409 & 152 (37) \\
\hline Antiplatelet & 409 & 159 (39) \\
\hline Anticoagulation & 409 & $28(7)$ \\
\hline Statin & 409 & $116(28)$ \\
\hline \multicolumn{3}{|l|}{ Cardiac biomarker levels at presentation } \\
\hline NT-proBNP pmol. $\mathrm{L}^{-1}$ & 405 & $62.5(53.7-72.7)$ \\
\hline \multicolumn{3}{|l|}{ Troponin T } \\
\hline Cohort $1 \mu \mathrm{g} \cdot \mathrm{L}^{-1}$ & 239 & $0.015(0.014-0.017)$ \\
\hline Cohort $2 \mathrm{ng} \cdot \mathrm{L}^{-1}$ & 162 & $18.3(16.2-20.7)$ \\
\hline \multicolumn{3}{|l|}{ Mortality } \\
\hline In-hospital & 401 & $17(4)$ \\
\hline 30-day & 401 & $29(7)$ \\
\hline 1-year & 401 & $82(20)$ \\
\hline 2-year & 401 & 133 (33) \\
\hline 5-year & 401 & $245(61)$ \\
\hline
\end{tabular}

Data are presented as geometric mean $(95 \% \mathrm{CI})$ or $\mathrm{n}(\%)$. Mortality data excluded eight patients who did not have both cardiac biomarkers measured on admission. BMI: body mass index; $\mathrm{FEV}_{1}$ : forced expiratory volume in $1 \mathrm{~s}$; NT-proBNP: N-terminal pro-B-type natriuretic peptide; $P_{\mathrm{aCO}_{2}}$ : arterial carbon dioxide tension; $P_{\mathrm{aO}_{2}}$ : arterial oxygen tension. ${ }^{\#}$ : blood $\mathrm{pH}<7.3$. 


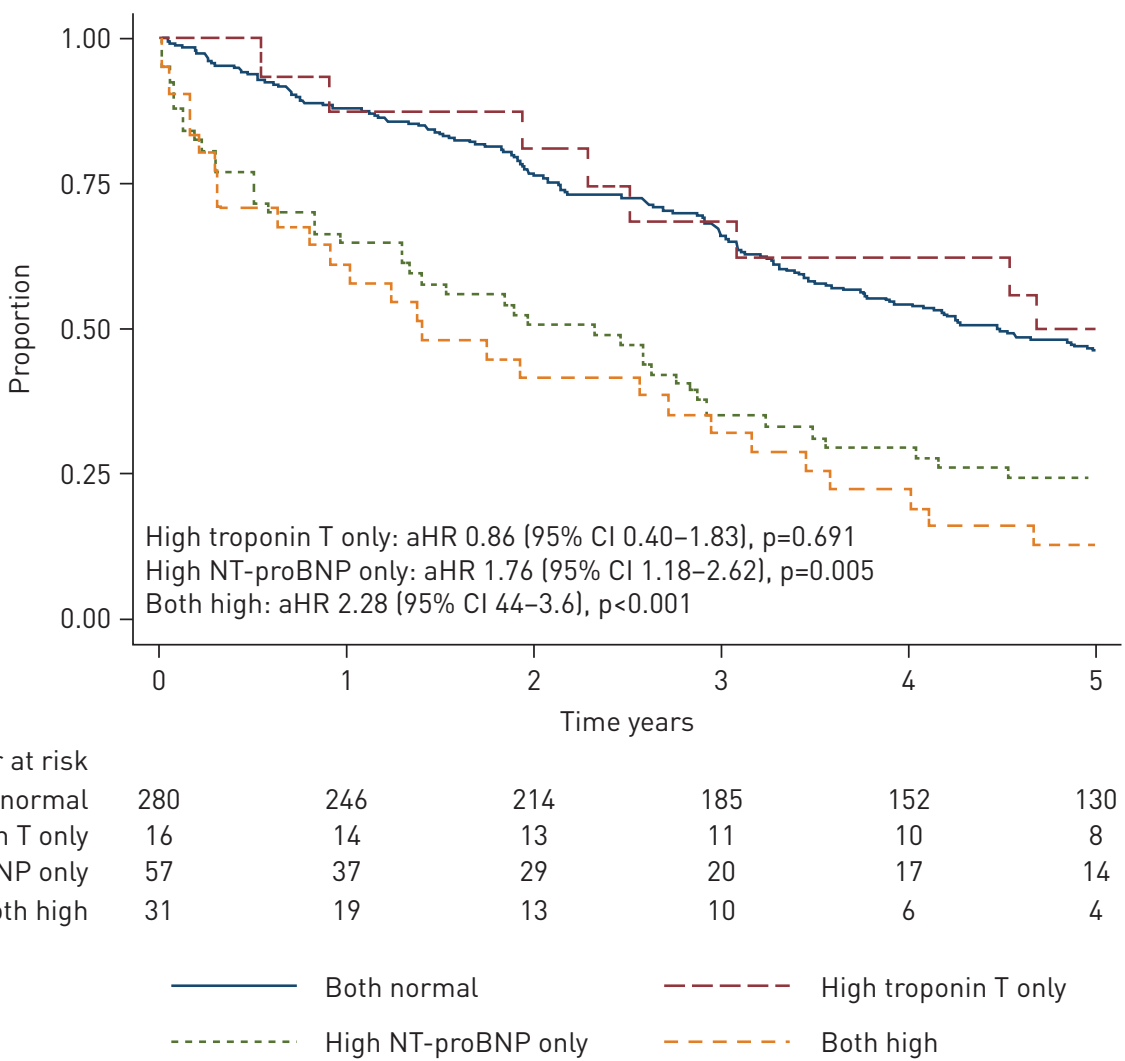

FIGURE 2 Kaplan-Meier curve of survival over 5 years after discharge for exacerbation of COPD. HR adjusted for CURB-65, acidaemia ( $\mathrm{pH}<7.30)$ and forced expiratory volume in $1 \mathrm{~s}(\%$ pred). aHR: adjusted hazard ratio; NT-proBNP: N-terminal pro-B-type natriuretic peptide.

severity (figure 2, table 2). Elevated troponin $\mathrm{T}$, however, did not predict long-term mortality. These findings suggest that subclinical cardiac dysfunction, but not necessarily acute cardiac injury, is an important determinant of long-term mortality in the setting of COPD exacerbations.

We have previously reported that elevated levels of NT-proBNP and troponin $\mathrm{T}$ and their combination were associated with a high short-term (30-day) mortality in cohort 1 [10]. In that analysis we did not find that either biomarker was associated with subsequent survival between 30 days and 1 year. This new

TABLE 2 Combined cohort adjusted and unadjusted hazard ratios (HRs) for 5-year survival, exacerbation-free survival and cardiac admission-free survival following discharge after index COPD admission according to cardiac biomarker status

\begin{tabular}{|c|c|c|c|c|c|c|}
\hline & \multicolumn{3}{|c|}{ Unadjusted } & \multicolumn{3}{|c|}{ Adjusted } \\
\hline Survival & 384 & & & 337 & & \\
\hline High troponin T only & & $0.89(0.43-1.80)$ & 0.738 & & $0.86(0.40-1.83)$ & 0.691 \\
\hline High NT-proBNP only & & $2.13(1.52-2.99)$ & $<0.001$ & & $1.76(1.18-2.62)$ & 0.005 \\
\hline Both high & & $2.82(1.87-4.26)$ & $<0.001$ & & $2.28(1.44-3.60)$ & $<0.001$ \\
\hline Exacerbation-free survival & 384 & & & 337 & & \\
\hline Both high & & $1.29(0.88-1.89)$ & 0.196 & & $1.27(0.83-1.95)$ & 0.277 \\
\hline Cardiac admission-free survival & 384 & & & 337 & & \\
\hline High troponin T only & & $0.87(0.44-1.70)$ & 0.681 & & $0.74(0.34-1.57)$ & 0.431 \\
\hline High NT-proBNP only & & $2.13(1.54-2.95)$ & $<0.001$ & & $1.75(1.20-2.55)$ & 0.003 \\
\hline Both high & & $2.55(1.70-3.84)$ & $<0.001$ & & $1.98(1.25-3.12)$ & 0.003 \\
\hline
\end{tabular}




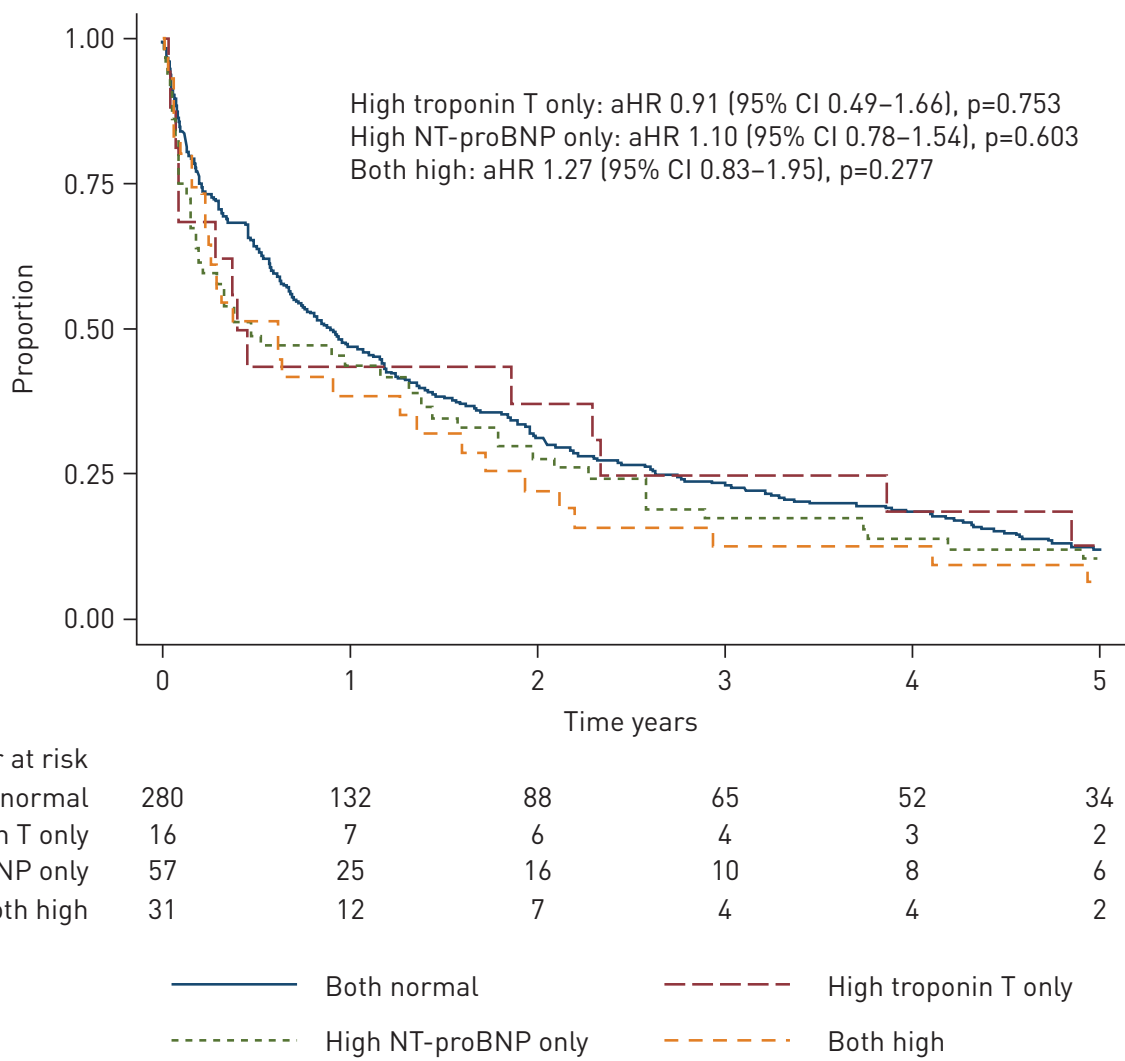

FIGURE 3 Kaplan-Meier curve of subsequent COPD hospitalisations following discharge after index COPD exacerbation. HR adjusted for CURB-65, acidaemia $(\mathrm{pH}<7.30)$ and forced expiratory volume in $1 \mathrm{~s}(\%$ pred). aHR: adjusted hazard ratio; NT-proBNP: N-terminal pro-B-type natriuretic peptide.

analysis extends these findings by combining data from two prospective cohorts with much longer follow-up. The findings show that elevated NT-proBNP is strongly associated with both short- and long-term prognosis after an exacerbation of COPD. NT-proBNP is released by the myocardium as a result of myocardial stretch and is a sensitive indicator of cardiac failure [15]. We also found that high levels of NT-proBNP predicted future admissions for cardiac disease but not future COPD admissions (figures 3 and 4). Hence, subclinical evidence of cardiac dysfunction at the time of an exacerbation may indicate poor cardiac reserve and be a harbinger of future cardiac events. Cardiac dysfunction did not predict acute COPD exacerbations suggesting that poor cardiac reserve may not be an aetiological factor in COPD exacerbations.

In the previous analyses of these cohorts $[10,12]$, a history of cardiac disease was not a predictor of 30-day or 1-year mortality. However, an analysis of this combined cohort shows that a history of cardiac disease and treatment with cardiac medications (notably diuretics) were associated with higher long-term mortality at 5 years (table S3). The associations of the biomarkers with 5 -year survival remained similar after adjustment for a history of cardiac disease, suggesting that acute cardiac dysfunction during exacerbations of COPD is associated with a poor long-term prognosis independently of a known cardiac disease.

While troponin $\mathrm{T}$ levels on admission were associated with short-term mortality in these cohorts and have been linked to worse prognosis in other studies, they did not predict long-term mortality, nor did they predict future cardiac admissions in these cohorts $[4,16]$. Cardiac troponins form part of the diagnosis of myocardial infarction but are sensitive markers of myocardial injury of any cause, including acute compromise of the pulmonary circulation [15-17]. Our observation that troponin is associated with short-term but not long-term mortality is probably best explained by troponins being released as a consequence of the acute COPD exacerbation causing transient cardiac myocyte injury, but this does not necessarily reflect clinically important underlying cardiac disease. There was no evidence that the raised levels of troponin $\mathrm{T}$ were caused by acute coronary syndromes in the study: those with clinically suspected acute coronary syndromes were excluded, and there was little electrocardiographic evidence of cardiac ischaemia. Having a raised troponin level does not necessarily mean an acute coronary syndrome, even among those with a known history of coronary artery disease, as the diagnosis requires a compatible 


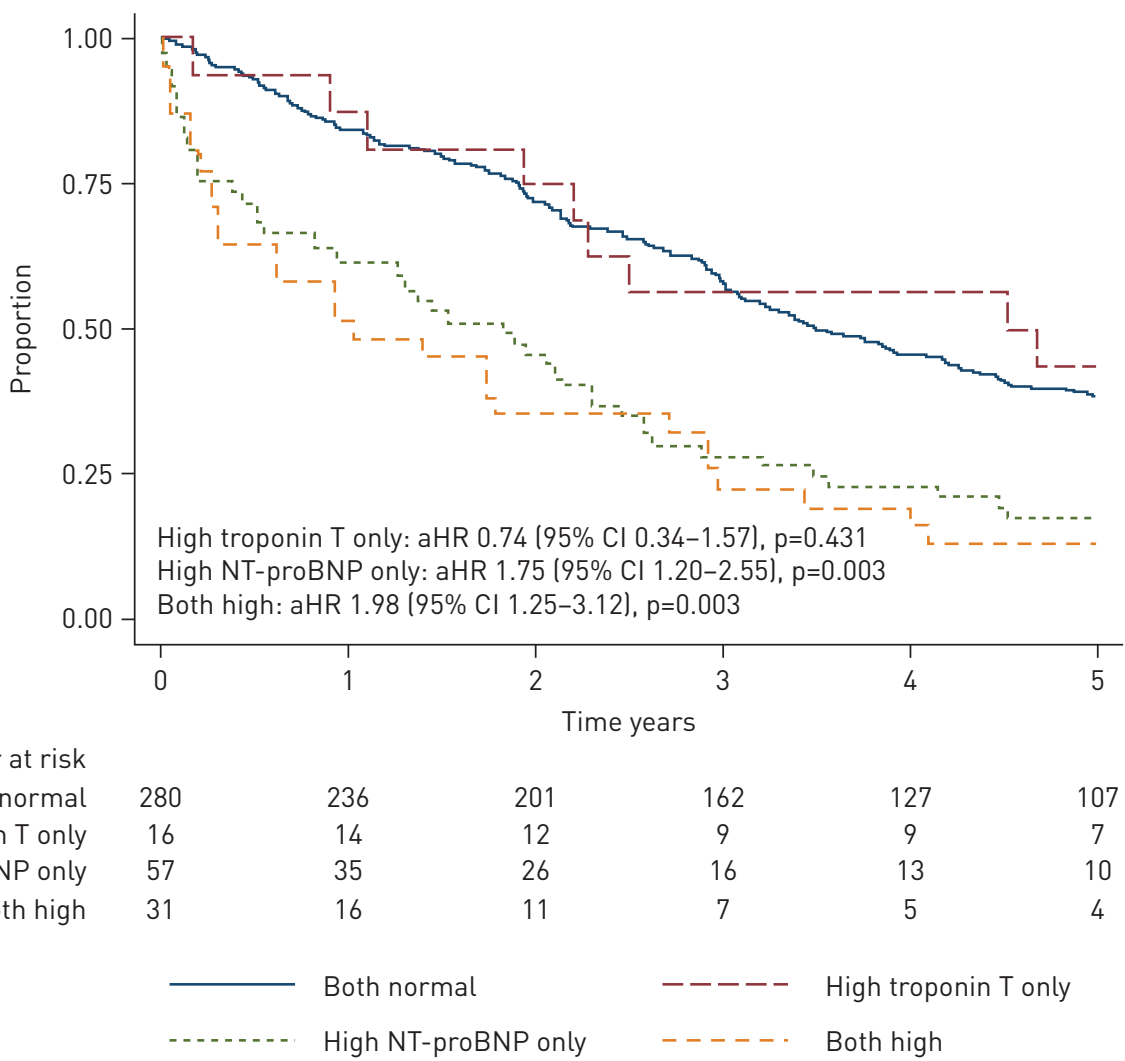

FIGURE 4 Kaplan-Meier curve of subsequent cardiac hospitalisations over 5 years following discharge after index COPD exacerbation. HR adjusted for CURB-65, acidaemia $(\mathrm{pH}<7.30)$ and forced expiratory volume in $1 \mathrm{~s}$ (\% pred). aHR: adjusted hazard ratio; NT-proBNP: N-terminal pro-B-type natriuretic peptide.

clinical history, serial changes of troponin and dynamic electrocardiographic changes. In most patients with raised troponins there is insufficient evidence to support the diagnosis of acute coronary syndrome [18], and we have previously shown that ECG and chest radiographs often do not detect subclinical biomarker derangements [13]. An additional analysis of these cohorts also revealed that a history of cardiac disease was not associated with a high troponin level on admission (OR 1.22, 95\% CI 0.69-2.14, p=0.499).

There is no universally accepted biological mechanism linking COPD and heart disease although there are several plausible explanations. COPD and cardiac disease share many risk factors such as smoking, older age and a chronic inflammatory state [19]. COPD is associated with atherosclerosis and arterial stiffness $[20,21]$, which increase the risk of cardiac ischaemia [22]. COPD exacerbations are associated with elevated systemic inflammatory markers such as C-reactive protein, interleukin-6 and platelet activation, which are also linked to atherosclerosis [23-26]. Our findings suggest that while an acute insult (i.e. the index admission for COPD) may lead to a rise in troponin levels, only those with cardiac remodelling and reduced cardiac reserve indicated by a high NT-proBNP have a worse long-term prognosis.

Exacerbations of COPD are known to increase systemic sympathetic tone leading to higher metabolic demands of tissues and increasing the risk of cardiac ischaemia [27]. Furthermore, severe exacerbations of COPD are usually treated with high doses of $\beta 2$ agonists, which stimulate cardiac $\beta 2$ receptors leading to increases in heart rate [28] and increasing cardiac stress.

Lung hyper-inflation caused by COPD is associated with reduced cardiac exercise tolerance so could contribute to cardiac comorbidity in COPD patients [29]. Pulmonary hypertension is estimated to affect around $30 \%$ of patients with moderate and severe COPD [30], causing right ventricular dilatation and hypertrophy, leading to restriction of the left ventricle resulting in a lower cardiac output [31].

Many of these inflammatory and physiological mechanisms are likely to worsen during exacerbations of COPD. We have previously found that NT-proBNP continues to rise during the first few days of a COPD exacerbation but falls as the patient recovers a few weeks later [11]. Whether NT-proBNP during stable COPD is an indicator of long-term prognosis is not yet known [32]. As up to half of COPD patients have left ventricular dysfunction, further investigations into this biochemical marker of cardiac overload may provide valuable insights into the complex interplay of heart-lung pathophysiology [33-35]. 
A major strength of this study is that these are large, unselected, prospectively recruited cohorts of patients with exacerbations of COPD, with near-complete follow-up data. The outcomes were objective (mortality or readmission). We were also able to adjust for confounders by the severity of the exacerbation and lung function impairment in the analyses. A limitation is that both cohorts are from a single centre; however, this reduces the potential for confounding. The overall 5-year mortality of $61 \%$ is similar to other cohorts [4], suggesting that our findings are likely to be generalisable to other centres.

There were some differences between the cohorts: cohort 2 was smaller and excluded patients with severe renal disease or terminal malignancy meaning that patients recruited into cohort 2 were healthier than cohort 1. Participants in cohort 2 were less likely to die in 5 years (HR 0.71, 95\% CI 0.54-0.93, p=0.013), but there was no difference between cohorts once adjusted for CURB-65, $\mathrm{pH}$ and $\mathrm{FEV}_{1} \%$ pred (adjusted HR $0.95,95 \%$ CI $0.70-1.29, \mathrm{p}=0.75$ ). The findings were similar for each cohort if analysed separately, hence it seems reasonable to combine the cohorts for the ease of interpretation. In both cohorts, the patients were treated according to best practice at time of admission, and participation in these studies did not influence treatment. The absolute number of patients with elevated biomarkers is also low, which might influence the results of analyses in particular those for troponins. Other limitations of this study include the possibility that some readmissions were not added to the electronic database if these occurred outside the Waikato region, although all deaths are recorded regardless of where they occurred. Another limitation is that although cardiac medications on admission were recorded, data on subsequent cardiac medications received during the follow-up period were not. Therefore, we were unable to analyse the impact of cardiac medications on long-term outcomes following an exacerbation of COPD. The frequency of COPD exacerbations before index admission was also not recorded and therefore was not accounted for in the analyses. A further limitation is that the treating clinicians were not blinded to the results of the cardiac biomarkers and that this information may have influenced their diagnosis of acute cardiac disease and management. This might be expected to reduce the association between biomarkers and mortality, but it is possible that knowledge of a previously abnormal NT-proBNP influenced clinicians to diagnose future events as being of cardiac origin, inflating the association between NT-proBNP and cardiac admissions. The exact causes of death were also not available, and we were unable to analyse whether they differed between biomarker groups.

These findings highlight the critical importance of cardiac comorbidity in COPD. This could be even more important at the time of a COPD exacerbation but may not be recognised. Clinicians should be aware of this and carefully look for cardiac involvement. Whether early diagnosis and treatment of cardiac disease in patients with COPD could improve both long- and short-term outcome is unproven, but identifying subclinical cardiac disease will likely help the assessment of prognosis. Future research is needed to better characterise the relationship and understand the mechanisms of cardiac dysfunction in COPD. It would be of particular interest to determine whether cardiac dysfunction is the cause of higher mortality in COPD or whether it is simply a marker of more severe COPD. Our finding that cardiac dysfunction during a COPD exacerbation is associated with future cardiac admissions but not future COPD admissions signals that treatments targeting cardiac dysfunction may improve outcomes in this population by reducing cardiac rather than respiratory events.

In summary, exacerbations of COPD have a poor long-term prognosis with more than half of patients dying within 5 years of hospitalisation. Abnormal levels of NT-proBNP, a marker of cardiac stretch and cardiac failure, at the time of the COPD admission are associated with higher long-term mortality and subsequent admissions for cardiac disease.

Acknowledgements: We would like to thank the study participants. We also thank Manisha Cooray, summer student for data collection.

Data availability: individual deidentified participant data will only be available if requested.

Support statement: This study was funded by a summer-studentship grant from the Waikato Medical Research Foundation. Initial recruitment and data collection for cohorts 1 (COPDEC) and 2 (BREATHE) were funded by the Waikato Respiratory Research Fund and the Waikato Medical Research Foundation. Funding information for this article has been deposited with the Crossref Funder Registry.

Conflict of interest: None declared.

References

1 Sin DD, Man SF. Chronic obstructive pulmonary disease as a risk factor for cardiovascular morbidity and mortality. Proc Am Thorac Soc 2005; 2: 8-11.

2 Zvezdin B, Milutinov S, Kojicic M, et al. A postmortem analysis of major causes of early death in patients hospitalized with COPD exacerbation. Chest 2009; 136: 376-380. 
3 Maclay JD, McAllister DA, Macnee W. Cardiovascular risk in chronic obstructive pulmonary disease. Respirology 2007; 12: 634-641.

4 MacDonald MI, Shafuddin E, King PT, et al. Cardiac dysfunction during exacerbations of chronic obstructive pulmonary disease. Lancet Respir Med 2016; 4: 138-148.

5 Dransfield MT, Voelker H, Bhatt SP, et al. Metoprolol for the prevention of acute exacerbations of COPD. $N$ Engl J Med 2019; 381: 2304-2314.

6 Piquet J, Chavaillon JM, David P, et al. French College of General Hospital Respiratory P. High-risk patients following hospitalisation for an acute exacerbation of COPD. Eur Respir J 2013; 42: 946-955.

7 Singanayagam A, Schembri S, Chalmers JD. Predictors of mortality in hospitalized adults with acute exacerbation of chronic obstructive pulmonary disease. Ann Am Thorac Soc 2013; 10: 81-89.

8 Guo Y, Zhang T, Wang Z, et al. Body mass index and mortality in chronic obstructive pulmonary disease: a dose-response meta-analysis. Medicine (Baltimore) 2016; 95: e4225.

9 Garcia-Aymerich J, Serra Pons I, Mannino DM, et al. Lung function impairment, COPD hospitalisations and subsequent mortality. Thorax 2011; 66: 585-590.

10 Chang CL, Robinson SC, Mills GD, et al. Biochemical markers of cardiac dysfunction predict mortality in acute exacerbations of COPD. Thorax 2011; 66: 764-768.

11 Shafuddin E, Chang CL, Cooray M, et al. Changes in biomarkers of cardiac dysfunction during exacerbations of chronic obstructive pulmonary disease. Respir Med 2018; 145: 192-199.

12 Shafuddin E, Chang C, Hancox R. Cardiac biomarkers and outcomes of COPD exacerbations. Eur Respir J 2019; 54: Suppl. 63, PA4290.

13 Shafuddin E, Chang CL, Cooray M, et al. Cardiac dysfunction in exacerbations of chronic obstructive pulmonary disease is often not detected by electrocardiogram and chest radiographs. Intern Med J 2019; 49: 761-769.

14 Lee MH, Chang CL, Davies AR, et al. Cardiac dysfunction and N-terminal pro-B-type natriuretic peptide in exacerbations of chronic obstructive pulmonary disease. Intern Med J 2013; 43: 595-598.

15 Ponikowski P, Voors AA, Anker SD, et al. 2016 ESC Guidelines for the diagnosis and treatment of acute and chronic heart failure: The Task Force for the diagnosis and treatment of acute and chronic heart failure of the European Society of Cardiology (ESC) developed with the special contribution of the Heart Failure Association (HFA) of the ESC. Eur Heart J 2016; 37: 2129-2200.

16 Pavasini R, d'Ascenzo F, Campo G, et al. Cardiac troponin elevation predicts all-cause mortality in patients with acute exacerbation of chronic obstructive pulmonary disease: systematic review and meta-analysis. Int J Cardiol 2015; 191: 187-193.

17 Chi SY, Kim EY, Ban HJ, et al. Plasma N-terminal pro-brain natriuretic peptide: a prognostic marker in patients with chronic obstructive pulmonary disease. Lung 2012; 190: 271-276.

18 Harvey MG, Hancox RJ. Elevation of cardiac troponins in exacerbation of chronic obstructive pulmonary disease. Emerg Med Australas 2004; 16: 212-215.

19 Hawkins NM, Virani S, Ceconi C. Heart failure and chronic obstructive pulmonary disease: the challenges facing physicians and health services. Eur Heart J 2013; 34: 2795-2803.

20 Sabit R, Bolton CE, Edwards PH, et al. Arterial stiffness and osteoporosis in chronic obstructive pulmonary disease. Am J Respir Crit Care Med 2007; 175: 1259-1265.

21 Lahousse L, van den Bouwhuijsen QJ, Loth DW, et al. Chronic obstructive pulmonary disease and lipid core carotid artery plaques in the elderly: the Rotterdam Study. Am J Respir Crit Care Med 2013; 187: 58-64.

22 Cecelja M, Chowienczyk P. Role of arterial stiffness in cardiovascular disease. JRSM Cardiovasc Dis 2012; 1 cvd.2012.012016.

23 Sabit R, Bolton CE, Fraser AG, et al. Sub-clinical left and right ventricular dysfunction in patients with COPD. Respir Med 2010; 104: 1171-1178.

24 Messner B, Bernhard D. Smoking and cardiovascular disease: mechanisms of endothelial dysfunction and early atherogenesis. Arterioscler Thromb Vasc Biol 2014; 34: 509-515.

25 Malerba M, Clini E, Malagola M, et al. Platelet activation as a novel mechanism of atherothrombotic risk in chronic obstructive pulmonary disease. Expert Rev Hematol 2013; 6: 475-483.

26 Ross R. Atherosclerosis - an inflammatory disease. N Engl J Med 1999; 340: 115-126.

27 Malpas SC. Sympathetic nervous system overactivity and its role in the development of cardiovascular disease. Physiol Rev 2010; 90: 513-557.

28 Cazzola M, Calzetta L, Matera MG. beta(2)-adrenoceptor agonists: current and future direction. Br J Pharmacol 2011; 163: 4-17.

29 Vassaux C, Torre-Bouscoulet L, Zeineldine S, et al. Effects of hyperinflation on the oxygen pulse as a marker of cardiac performance in COPD. Eur Respir J 2008; 32: 1275-1282.

30 Elwing J, Panos RJ. Pulmonary hypertension associated with COPD. Int J Chron Obstruct Pulmon Dis 2008; 3 : $55-70$.

31 Boussuges A, Pinet C, Molenat F, et al. Left atrial and ventricular filling in chronic obstructive pulmonary disease An echocardiographic and Doppler study. Am J Respir Crit Care Med 2000; 162: 2 Pt. 1, 670-675.

32 Gale CP, White JE, Hunter A, et al. Predicting mortality and hospital admission in patients with COPD: significance of NT pro-BNP, clinical and echocardiographic assessment. J Cardiovasc Med (Hagerstown) 2011; 12 613-618

33 Macchia A, Rodriguez Moncalvo JJ, Kleinert M, et al. Unrecognised ventricular dysfunction in COPD. Eur Respir J 2012; 39: 51-58.

34 Agarwal SK, Heiss G, Barr RG, et al. Airflow obstruction, lung function, and risk of incident heart failure: the Atherosclerosis Risk in Communities (ARIC) study. Eur J Heart Fail 2012; 14: 414-422.

35 Rutten FH, Cramer MJ, Lammers JW, et al. Heart failure and chronic obstructive pulmonary disease: an ignored combination? Eur J Heart Fail 2006; 8: 706-711. 